\title{
La Rationalisation du jeu
}

Une Théorie critique du jeu numérique

Sara M. Grimes and Andrew Feenberg

Translator. Marion Delage de Luget

(2) OpenEdition

Journals

Electronic version

URL: http://journals.openedition.org/appareil/1127

DOI: 10.4000/appareil.1127

ISSN: 2101-0714

Publisher

MSH Paris Nord

Electronic reference

Sara M. Grimes and Andrew Feenberg, « La Rationalisation du jeu », Appareil [Online], Articles, Online since 11 September 2013, connection on 30 July 2020. URL : http://journals.openedition.org/appareil/ 1127 ; DOI : https://doi.org/10.4000/appareil.1127

This text was automatically generated on 30 July 2020 .

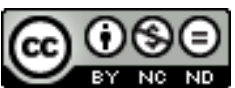

Appareil est mis à disposition selon les termes de la Licence Creative Commons Attribution - Pas d'Utilisation Commerciale - Pas de Modification 4.0 International. 


\title{
La Rationalisation du jeu
}

\author{
Une Théorie critique du jeu numérique \\ Sara M. Grimes and Andrew Feenberg \\ Translation : Marion Delage de Luget
}

1 Les jeux numériques sont l'une des activités de loisirs les plus dynamiques de ce nouveau siècle. Partant du statut de passe-temps pour enfants marginalisés, ils se sont rapidement développés en une industrie mondiale pesant plusieurs milliards de dollars. Selon de récentes estimations, le marché mondial des jeux numériques a généré 41.9 milliards de dollars de ventes en 2007 et devrait dépasser 68 milliards de dollars d'ici 2012 (Bond, 2008). La société d'analyse ComScore Media Matrix estime qu'environ 217 millions de personnes à travers le monde ont joué à des jeux en ligne en 2007 - un nombre qui continue de se multiplier à mesure que l'accès Internet à haut débit se répand à travers l'Asie et d'autres régions (Castronova, 2005). En conséquence, l'attention des universitaires aux jeux numériques s'est elle aussi sensiblement accrue ces dernières années, alors que les chercheurs s'efforcent de comprendre tant le phénomène que l'essor industriel qu'il a généré.

2 Bien qu'un certain nombre de théories des jeux numériques aient maintenant émergé, et ce à partir de perspectives variées, les applications de la théorie critique à l'étude des jeux numériques en sont encore au stade préliminaire. À quelques exceptions près (Kirkpatrick, 2008), les travaux existants dans ce domaine (y compris Postigo, 2003; de Peuter et Dyer-Witheford, 2005; Taylor, 2006a; Grimes, 2006) se sont concentrés principalement sur l'expansion des processus de production englobant les joueurs euxmêmes (comme la main d'œuvre, la commercialisation, etc.), reproduisant la même opposition binaire entre les notions de travail et de jeu qui a longtemps caractérisé les travaux critiques sur le jeu et les loisirs (Gruneau, 1999). D'autres contributions, comme celles de Kline, de Peuter et le Dyer-Witheford (2003), Brookey et Booth (2006) et Taylor (2006b, 2006c), ont mis l'accent sur la façon dont les limitations structurelles des jeux numériques (qu'elles soient commerciales, sociales ou technologiques) impactent à la fois sur les possibilités d'interaction et sur la capacité que le joueur a d'agir en tant que sujet (player agency). Mais ces contributions ne sont pas parvenues à refaire le lien entre ces limitations et le jeu lui-même. À ce jour, très peu d'attention a été accordée à la 
formulation d'une théorie critique des jeux numériques qui permettrait une plus grande compréhension de la façon dont les pratiques du jeu (play) elles-mêmes reproduisent les processus plus larges de la rationalisation du travail au sein des sociétés capitalistes modernes.

Pourtant, tout laisse à penser que la pratique des jeux numériques - surtout dans le cas des jeux en ligne massivement multijoueurs (MMOG) - soit un exemple particulièrement adapté pour une application plus large de l'analyse critique. Selon l'argument de Feenberg, les jeux (games) «illustrent formellement les systèmes rationnels » $(1995$, p . 193). Les jeux (games) se détachent de l'action communicative indifférenciée de la vie « ordinaire" pour imposer une forme rationnelle sur un secteur d'expérience (Habermas, 1984), de la même façon que les marchés économiques, les systèmes juridiques et la recherche scientifique. Les règles définissent un domaine de jeu (play domain) avec des mesures sans équivoque de succès et d'échec, et une distinction nette entre les actions stratégiques et non stratégiques. Dès lors qu'on y ajoute la médiation technique et le mercantilisme, les jeux (games) deviennent une base pour la production d'une forme d'« ordre institutionnel » analysable dans des termes semblables à ceux employés dans l'étude d'autres systèmes de rationalité sociale (Weber, 1958 ; Henricks, 2006). Parce qu'ils relèvent de cette médiation technique, et qu'ils sont des systèmes commerciaux à travers lesquels d'importantes populations de joueurs se réunissent pour se livrer à une interaction sociale organisée, les jeux en ligne massivement multijoueurs (MMOGs) fournissent un cas d'étude idéal pour explorer la relation entre les jeux et la rationalité sociale.

4 Le terme "rationalité sociale » est utilisé ici dans un sens purement descriptif pour référer à des pratiques organisationnelles qui s'apparentent à des cas de paradigme de la rationalité, comme les sciences et les mathématiques. Trois types de pratiques satisfont à cette condition: (1) l'échange d'équivalents; (2) la classification et l'application des règles; et (3) l'optimisation de l'effort et le calcul des résultats. Nous n'avons pas l'intention d'impliquer que les pratiques qui diffèrent de ce que nous appelons «la rationalité sociale» soient irrationnelles, nous ne prétendons pas non plus que seuls la science et les mathématiques soient rationnels, dans une acception large du terme. Les pratiques correspondant aux trois principes précédemment cités figurent dans toutes les sociétés, sous formes individuelles ou culturelles. Par exemple, un match de football entre amis suit des règles, mais ce n'est pas une forme d'ordre social imposé par une organisation à grande échelle, aussi n'est il pas, selon notre acception, considéré comme un cas de rationalité sociale. De même, une coutume tribale sanctionnant le respect des biens d'autrui ou guidant la production artisanale peut être rationnelle dans le sens où elle accroît les chances de survie de la communauté ; mais si elle n'est pas imposée consciemment, si elle est tout simplement héritée du passé, ceci non plus n'est pas socialement rationnel. Le differentia specifica de la rationalité sociale est le rôle des trois principes de la pratique rationnelle dans les organisations sociales et les systèmes d'interactions régulées par les médias, ce rôle qui, sur une grande échelle, au moins, appartient uniquement à la modernité (Feenberg, 2008). (Note 1)

5 Pour mener à bien l'étude de la rationalité sociale, la théorie d'instrumentalisation de Feenberg offre un point d'entrée unique. La théorie de l'instrumentalisation a été initiée pour analyser la technologie à deux niveaux : l'instrumentalisation primaire, qui décrit comment «les fonctions sont séparées du continuum de la vie quotidienne et des sujets sont mis dans une position à partir de laquelle ils peuvent agir sur eux», et 
l'instrumentalisation secondaire, qui met l'accent sur les aspects sociaux, culturels, et les forces politiques qui influent sur les choix de conception (de mise en forme) de ces fonctions en tant qu'elles sont réalisées dans des dispositifs et des systèmes (Feenberg, 1999, p. 202). Ces deux instrumentalisations sont des catégories analytiques qui sont utiles à la compréhension de la bilatéralité des artefacts techniques, qui sont à la fois techniquement rationnels et significatifs du point de vue socioculturel.

Bien que la théorie de l'instrumentalisation ait été initialement conçue comme une structure aidant à comprendre la technique, l'approche proposée s'étend aussi à d'autres systèmes de rationalité sociale. Comme Feenberg l'explique : «Tous les systèmes rationnels ont ce double aspect: d'une part, ils sont une structure d'opération basée sur un ou plusieurs des trois principes de la rationalité sociale et, d'autre part, ils sont un monde complexe expérimenté par ceux qui s'y inscrivent » (2008). (Note 2)

7 Comme les jeux (games) deviennent de plus en plus rationnels grâce au contrôle des entreprises et à la technologisation, les caractéristiques rationnelles fondamentales communes à tous les jeux formels prennent une importance inattendue. L'échange de coups entre les joueurs qui sont encore à égalité au début de la partie correspond au premier principe. Les règles strictes et les stratégies illustrent le deuxième et le troisième principe. Les jeux en ligne massivement multijoueurs imposent ces trois types de pratique rationnelle comme suit: les joueurs et les mouvements de joueurs sont normalisés par le code du programme (échange d'équivalents), les règles officielles sont établies par le moteur du jeu et les exploitants ainsi que la communauté des joueurs (classification et application de règles) ; et les efforts des joueurs sont optimisés et calculés par le biais de mises à niveau numériques et de systèmes de points qui sont encore renforcés par le statut et le capital social octroyés aux joueurs de haut standing (optimisation de l'effort et calcul des résultats).

8 Dans le même temps, cependant, les jeux en ligne massivement multijoueurs sont constitués par une expérience de jeu (play experience) basée sur la collaboration qui s'étend au-delà de ces systèmes rationnels. Comparable au carnaval de Bakhtin, les jeux en ligne massivement multijoueurs sont caractérisés par un type d' "action symbolique qui est rarement simplement cantonnée au jeu: elle articule des significations culturelles et politiques » (Stallybrass et White, 1986, p. 43). Comme Taylor (2002) le décrit, les joueurs de jeux en ligne massivement multijoueurs investissent un temps considérable dans des réunions, dans la création de contenu, produisant des cultures et des communautés, partageant des activités de loisirs, et transgressant les limites de la partie. Ces joueurs possèdent un haut niveau de connaissances quant au sujet, ce qui leur permet de prendre part à la technologie des jeux numériques de façon imprévue, et cela a un impact énorme sur le développement, le contenu et le fonctionnement des jeux (games) dans la culture numérique. Ainsi, on peut aussi comprendre les jeux en ligne massivement multijoueurs comme un lieu où les joueurs et les entreprises de développement de jeux (games) s'affrontent quant à la conception et l'utilisation des environnements de jeu (game environments), et quant à leur contenu.

9 La théorie critique offre un point d'entrée unique à cet égard, un point de vue qui intègre la critique du capitalisme de Marx et la critique de Weber sur la rationalité, et propose, à partir de là, d'élargir le champ de réflexion. En situant les technologies dans les contextes sociaux, institutionnels et idéologiques dans lesquels elles sont apparues, et dans lesquels elles évoluent, la théorie critique aborde à la fois les relations symbiotiques qui existent entre le technique et le social, et la menace spécifique de la 
technocratie dans les sociétés modernes. De cette façon, la théorie critique permet un examen plus approfondi de la manière dont les jeux (games) s'acquittent de fonctions multiples, à la fois pour leurs propriétaires et pour les joueurs. Nous proposons une telle approche par le biais d'une adaptation de la théorie critique de la technologie de Feenberg ; c'est par l'application de ses concepts d'instrumentalisation et de rationalité sociale que s'élabore une théorie du jeu (play) novatrice, le comparant à un processus rationalisé de la modernité. Cette théorie de la « ludification " procure un ensemble de critères d'évaluation des jeux rationalisés (rationalized games) en utilisant une approche à deux niveaux, qui tient compte à la fois de la façon - ou des façons - dont une partie (un jeu - game) est engagée dans des types de pratiques rationnelles, mais aussi des conditions sociales, culturelles et politiques dans lesquelles les joueurs d'un jeu (game) se l'approprient et le contestent.

Cet article explique en détail la théorie de la ludification, ainsi que cette notion corollaire : celle des jeux (games) compris comme des systèmes de rationalité sociale. La discussion est suivie d'une étude de cas de World of Warcraft (WoW), un jeu en ligne massivement multijoueurs très populaire qui bénéficie présentement de plus 10 millions de joueurs à travers le monde (Blizzard Entertainment, Inc, 2008). Le but de cet article est de fournir un cadre pour découvrir les propriétés rationnelles des jeux en ligne massivement multijoueurs, et, plus largement, de situer les jeux numériques au sein de la tendance socio-historique de rationalisation qui continue à façonner les pratiques de jeu (play) modernes. Notre intention n'est pas ici de montrer que ces jeux rationalisés sont qualitativement «meilleurs » ou "pires » que les formes de jeux non rationalisés, mais plutôt de susciter le débat autour de l'impact et de la signification de la rationalisation sur les paramètres du jeu (play), ses pratiques et l'expérience qu'on peut en avoir.

\section{Les jeux comme systèmes de rationalité sociale}

Même si les notions romantiques d'un « jeu à l'état pur » et de " jouer pour l'amour du jeu» continuent d'influencer les notions contemporaines de loisirs (Sutton-Smith, 1997), les théoriciens critiques ont depuis longtemps mis en évidence le rôle crucial que le jeu (play) remplit au sein du capitalisme avancé. D’une part, le loisir est intégré dans le cycle de travail, qui requiert et organise les périodes de repos et de récupération entre les efforts productifs (Marcuse, 1969). D'autre part, la marchandisation croissante des loisirs dans la culture de grande consommation brouille les lignes entre jeu et consommation (Bourdieu, 1991 ; Rifkin, 2000). Un certain nombre de théoriciens du jeu (play) prétendent que les sphères du travail et du jeu (play), si jamais elles avaient jamais été séparées, sont maintenant inextricablement enchevêtrées (Huizinga, 1955 ; Lasch, 1979). Cette intrication est essentiellement perçue en termes d'assimilation du jeu (play) et des loisirs dans les domaines rationnels de la production et de la consommation, mais elle est également comprise en termes de diffusion d'une certaine dose d'humeur ludique dans le processus de travail post industriel (Turner, 1974 ; Hans, 1981 ; de Certeau, 1984). Ainsi, bien que le jeu et les autres formes de loisirs soient souvent décrits dans les théories du jeu (play) comme extra-économiques - remplissant une fonction avant tout spirituelle, sociale, ou des fonctions cognitives -, leur pratique effective est de plus en plus appréhendée en ce qu'elle survient dans un contexte de processus socio-économiques complexes. 
12 La relation entre la production et les loisirs reste un axe essentiel dans les débats actuels sur la marchandisation et l'instrumentalisation du jeu (play), notamment dans les études récentes sur les jeux numériques massivement multijoueurs. Par exemple, la monétisation de l'économie du jeu (game) virtuel (qui apparut tout d'abord sous la forme d'un échange informel, un joueur échangeant un des objets du jeu - un item contre de la monnaie du monde réel, et s'est depuis étendue à toute une variété de modèles de revenus, plus ou moins officiels et plus ou moins sanctionnés) est souvent décrite en termes de travail (Grimes, 2006, 2008). Taylor décrit l'effort des joueurs de jeux en ligne massivement multijoueurs pour «insuffler à [leur personnage de jeu, leur avatar] des qualités, leur procurer un statut social, des talents » (2002, p. 232) comme un type de travail, et une collaboration à la paternité du jeu (game). D'autres, comme Postigo (2003), de Peuter et Dyer-Witheford (2005), Kücklich (2005), et Coleman et DyerWitheford (2007), ont exploré la façon dont des pratiques telles que le piratage ou le modding ${ }^{1}$ sont devenues les sources principales du travail immatériel, et contribuent souvent directement au cycle de développement des jeux numériques.

En tant que système d'organisation dominant d'une proportion croissante de notre expérience de la vie quotidienne, la notion de production est évidemment devenue un point focal essentiel dans les discussions rapprochant le jeu de la modernité (Gruneau, 1999). Pour que les activités de jeu (play) deviennent plus « organisées, même administrées »(Marcuse, 1969, p. 32), elles sont de plus en plus structurées par les mêmes valeurs, les mêmes priorités, les mêmes compétences et les mêmes normes que celles qui commandent la journée de travail (Mills, 1956; Bourdieu, 1978). Cependant, l'accent mis sur la relation entre le travail et le jeu (play) néglige un aspect clé du processus de rationalisation, à savoir qu'il se déroule différemment dans différents contextes institutionnels (Henricks, 2006). Au lieu de ne voir le jeu (play) que comme une victime des avancées économiques, il peut être plus utile d'étudier comment les jeux (games) eux-mêmes viennent afficher ces mêmes caractéristiques de la rationalisation qu'arborent les institutions qui imposent de l'ordre social et du contrôle.

14 À cet égard, les jeux (games) d'aujourd'hui seraient en retard quant aux processus de modernisation, puisque ceux-ci ont déjà intégré un large éventail de comportements génériques de l'homme dans le processus de rationalisation grâce à la technologie, aux marchés et au système juridique. Le jeu (play) lui aussi est maintenant de plus en plus recontextualisé comme un fondement de la société moderne à travers la marchandisation et la technologisation. Le jeu rationalisé (rationalized play) est ainsi non seulement congruent au grand récit de modernité, mais il fonctionne aussi comme une pratique sociale, reproduisant encore la rationalisation dans une autre facette de la vie quotidienne. Prenons ici un exemple chez Henricks, qui affirme: "[Le jeu (play)] expose des structures sociales seulement quelque peu différentes de celles trouvées dans d'autres parties de la vie. Ces structures non seulement limitent la liberté personnelle, mais elles permettent aussi aux gens d'accomplir des choses qu'ils ne pourraient pas faire seuls. [...] Jouer avec les autres, c'est entrer dans un domaine d'interconnexion qui est beaucoup plus complexe que le jeu (play) des particuliers avec le monde matériel. » (2006, p. 8-9)

Dans leur forme non rationalisée, les jeux (games) ne fonctionnent pas comme des systèmes de rationalité sociale, ils ne sont pas institutionnalisés sur une grande échelle, et donc ne génèrent pas de l'ordre social. Ceci évolue, cependant, avec l'incorporation de jeux (games) dans le commerce et la technologie. La professionnalisation du sport représente un point critique dans la transition des jeux (games) non rationalisés aux 
jeux (games) rationalisés (voir Lash, 1979; Bourdieu, 1991; Gruneau, 1999). La normalisation dans les sports organisés et les clubs de jeux va de paire avec une commercialisation de la fonction du spectateur, parce qu'elle transforme les joueurs et les mouvements des joueurs en unités prévisibles et mesurables. La jouabilité (gameplay) peut désormais être évaluée en fonction des critères fixés par des règles formelles strictes afin de créer une expérience homogène pour chaque participant. Cette expérience peut alors être réifiée en conformité avec les droits de diffusion, avec les parts d'audience, ainsi qu'avec les exigences de la culture de consommation de masse.

Cela commence par la professionnalisation des ligues sportives : la médiation technique (sous la forme de la technologie des médias, par exemple) et la rationalisation sociale ouvrent un jeu (game) donné aux processus de marchandisation. Dans certains cas, un jeu (game) joué par une communauté de joueurs non rémunérés pourrait devenir le terrain de recrutement pour une communauté de professionnels rémunérés, qui donneraient des représentations devant un public de spectateurs. Dans d'autres, les items ${ }^{2}$ du jeu (game) peuvent acquérir une valeur d'échange dans le monde réel. Dans chaque cas, cependant, la marchandisation de masse d'un jeu (game) sera précédée par sa standardisation et sa rationalisation. Comme Mosco le dit, la marchandisation est grandement facilitée par l'expansion de «la possibilité de mesurer et de surveiller, d'emballer et de reconditionner» (2004, p. 156) - des possibilités qui sont offertes par la médiation technique.

Dans le cas des sports avec spectateurs, toutefois, le contrôle des conditions de jeu (play) affecte beaucoup plus profondément les joueurs que l'auditoire. Lorsque la séparation entre spectateurs et joueurs disparaît, comme c'est le cas dans les jeux en ligne massivement multijoueurs, et quand les règles et les limites d'un jeu (game) ne reposent que sur leur médiation technique, les participants au jeu (game) sont intégrés dans sa conception. Potentiellement, cela réduit considérablement tout type de négociation spontanée et d'exceptions aux règles qui sont une part possible (et souhaitable) de la jouabilité quand un jeu (game) se joue sur une base individuelle, par exemple entre amis, sur un terrain de jeu local (Schwartzman, 1978; Hughes, 1995 ; Sutton-Smith, 1997). Au lieu de cela, dans un jeu (game) relevant d'une médiation technique, les actions des joueurs sont réduites à un ensemble prédéterminé de possibilités. Comme les jeux (games) et le jeu (play) sont transformés en un ensemble de plus en plus rationalisé d'activités, impliquant des populations énormes, et ce pour de longues périodes, ils institutionnalisent une forme d'ordre social. La masse des spectateurs-joueurs est désormais ordonnée par la technologie du jeu (play), comme les marchés ordonnent les consommateurs, les bureaucraties étatiques ordonnent les citoyens, et la technologie de production ordonne les travailleurs.

Les jeux (games) sont transformés en sources d'ordre social par l'incorporation de leurs aspects rationnels dans des stratégies organisationnelles, tant technologiques que commerciales. La jouabilité (et le joueur) est structurée et rationalisée par le jeu (game) lui-même, qui, notamment, prévoit (et souvent applique) la réglementation à laquelle ses joueurs doivent souscrire. Comme cette forme de jeu (play) est mise en œuvre à une échelle de plus en plus large, à travers différents types de jeux (games) et de formes de loisirs, son importance sociale s'accroît. Les joueurs eux-mêmes commencent à remplir un rôle crucial dans le fonctionnement du jeu (game) comme un système à grande échelle. Ce qui rend pour part ces jeux (games) attrayants pour les autres joueurs, c'est 
leur capacité à offrir une grande dynamique sociale via une expérience de jeu partagée (shared game play experience). De cette façon, les joueurs se transforment en une ressource qui maintient le fonctionnement du jeu (game) tel qu'il a été prévu, et légitime la valeur d'échange du jeu (game) comme une expérience sociale «conditionnée ». Ce processus est typique des systèmes de rationalité sociale, où même les êtres humains commencent à apparaître comme des porteurs d'éléments techniques, disponibles pour la manipulation par les entreprises techniques (Feenberg, 2008).

La caractéristique essentielle de la rationalisation est la capture d'activités quotidiennes par les entreprises et les médias. Cela inclue les jeux (games) ordinaires, qui ont toujours eu des qualités rationnelles (telles que des règles, des systèmes de points, des équipements normalisés, des ligues et des associations). De tels comportements sont aussi présents dans de nombreuses autres activités. Ils exemplifient à petite échelle les pratiques rationnelles de l'optimisation, l'échange des équivalents, la classification et l'application des règles; mais, jusqu'à l'époque moderne, il n'y avait pas d'entreprises capables de structurer les sociétés autour de tels comportements. Cette transformation s'est produite lorsque les caractéristiques rationnelles des pratiques quotidiennes sont devenues la base des systèmes techniques, économiques et juridiques, et aussi celle des entreprises dans les sociétés modernes. Les entreprises et les médias intègrent ces caractéristiques dans les structures bureaucratiques, commerciales et techniques, qui multiplient leur capacité et leur influence.

Dans ce contexte théorique, les systèmes de rationalité sociale devraient être conçus comme des agents actifs. De la même façon, cependant, leurs membres peuvent être plus ou moins conciliants dans l'accomplissement de fonctions au sein de la structure qu'ils conçoivent. La logique des entreprises et des médias est donc relativement indépendante des personnes qui y participent, mais, corrélativement, ces personnes ont une certaine indépendance qui se manifeste dans des actions qui induisent le changement, le pillage, l'intégration d'alternatives, subrepticement, là où un manque d'attention ou un faille du système de contrôle le permet, et ainsi de suite.

21 Malgré le niveau élevé de rationalisation qu'ont permis la médiation technique et la commercialisation, certains résultats imprévisibles restent donc non seulement possibles mais probables. Peu importe le niveau de rationalisation du jeu (game), ses joueurs restent engagés dans une lutte pour s'approprier leur pratique du jeu (play) et la signifier dans les contextes de leurs vies quotidiennes. Toutes les réponses ne se conforment pas à l'intention de rationaliser inscrite dans les modalités formalisées du jeu (game), et les comportements des joueurs peuvent souvent résister ou même défier l'ordre social sous-jacent. Cela comprend les interventions techniques spécialisées, telles que le piratage et le modding, ainsi que ces autres pratiques généralisées chez les joueurs comme la tricherie, les appropriations technologiques, les lectures subversives, les relations interpersonnelles, et la production de jeux (games) formalisés, de " paratextes» (tels que les fanfictions ${ }^{3}$, les " soluces ${ }^{4} »$, etc.) (Consalvo, 2007). Lorsque ces défis restructurent efficacement les aspects du jeu autour de la demande des joueurs, nous pouvons parler d'une "rationalisation démocratique ", en opposition à la rationalisation imposées par les entreprises officiellement propriétaires (Feenberg, 1999).

22 À bien des égards, tous les jeux (games) dépendent en dernier ressort de la participation et de l'adhésion des joueurs, qui s'engagent volontairement dans l'acte de jouer et, par 
voie de consensus et de collaboration, formulent les paramètres, les fictions et les fantasmes de l'expérience du jeu (play). Peu importe jusqu'à quel point on peut appliquer strictement les règles d'un jeu (game), la raison qui fait que l'on joue à un jeu (game), comme Geertz le fait valoir, est "que cela produit un commentaire méta social», une histoire que les joueurs «se racontent sur eux-mêmes » (1973). Alors que l'idée que le jeu (play) soit généré par les joueurs d'un jeu (game) puisse, à première vue, sembler en contradiction avec notre conception des jeux (games) comme des systèmes rationnels, nous pensons que c'est dans cette tension même entre liberté et contrainte, entre volontarisme et déterminisme, que les jeux (games) se présentent comme une forme de pratique sociale (Gruneau, 1999) et qu'ils viennent à fonctionner comme les systèmes de l'ordre social.

\section{Des règles à la ludification}

La rationalisation du jeu (play) s'appuie sur des ressources qui apparaissent au cours de la transition menant des activités de jeu (play) informelles à des jeux (games) organisés. Les débats relatifs à cette transition apparaissent tout au long des études fondamentales sur le jeu (play), souvent dans cette distinction posée entre le jeu (play) et les jeux (games). Une grande partie des premiers travaux dans ce domaine avalise ce que Sutton-Smith (1997) décrit comme une idéologie du «jeu (play) comme un progrès », reliant les caractéristiques rationnelles des jeux (games) (tels que les règles formelles et les paramètres) à la compréhension fonctionnaliste du jeu (play). Par exemple, Huizinga fait valoir que l'une des principales caractéristiques du jeu (play) est qu'il "exige un ordre absolu et suprême. Le moindre écart par rapport à cet ordre "gâche le jeu (game)", le prive de son caractère et lui ôte toute valeur " $(1955$, p. 10). Le jeu (play) apporte une perfection temporaire et limitée dans l'imparfaite confusion de la vie quotidienne, créant une situation «exceptionnelle » qui favorise la culture, ainsi que la formation de groupes sociaux.

C'est dans la classification hiérarchique des jeux (games) de Caillois que nous trouvons la formulation la plus claire (et la célébration) de la transition du jeu (play) libre au jeu (play) formel (avec règles obligatoires), décrite en termes d'une « progression par ordre de rang " qui se déplace le long d'« un continuum entre deux pôles opposés » (2001, p. 13). Le premier pôle, appelé paidia, décrit les formes de jeu (play) qui représentent la fantaisie illimitée et le jeu de rôle (role-play), les formes de divertissements improvisés et non scénarisés. Au pôle opposé, appelé ludus, "cette exubérance espiègle et impulsive est presque entièrement absorbée ou disciplinée par une tendance complémentaire, et à certains égards inverse [...] pour associer au jeu (play) des conventions arbitraires, impératives, et rendues fastidieuses à dessein » (p. 13). Caillois fait valoir que, à mesure que les sociétés se modernisent, le jeu (play) est de plus en plus caractérisé par ce pôle appelé ludus, progressant "de la turbulence vers les règles", et prenant forme à travers les " conventions, les techniques et les ustensiles " (p.29) des jeux rationalisés (rationalized games). Il soutient que, comme les règles et les jeux (games) sont institutionnalisés, le jeu se transforme " en l'instrument d'une culture féconde et décisive. »

Toutefois, les théoriciens qui l'ont suivi ont contesté ces idéalisations précoces des jeux organisés (organized games), des structures de la règle et du jeu téléologique (purposive play). Ils mettent plutôt en avant la relation dialectique qui existe entre les règles du jeu et le jeu (game rules and game play), "entre les possibilités socialement structurées et la 
capacité d'agir propre à l'Homme (human agency)» (Gruneau, 1999, p. 27). Par exemple, les sociologues qui étudient de nombreux sports et loisirs tendent à dire que nous approchons le jeu (play) au niveau de sa fonction de représentation - comme un texte culturel (Geertz, 1973), comme une structure de méta-communication (Bateson, 1973), ou en termes d'action symboliques ou "rhétoriques" (Sutton-Smith, 1997). Les études sur les jeux numériques ont de la même façon tenté d'aborder la dimension dialectique du jeu (game play), qui est de plus en plus envisagée comme une sorte de dialogue continu qui se produit entre le système d'un jeu (game) (le code du programme, les règles, l'interface graphique [Graphique User Interface]) et ses acteurs (Kirkpatrick, 2008). Par exemple, Salen et Zimmerman décrivent le "jeu significatif (meaningful play)» comme émergent « de la relation entre l'action de jeu (play action) et les résultats du système; c'est le processus par lequel un joueur prend des mesures au sein du système d'un jeu (game), et le système répond à l'action » (2003, p. 34).

Il est important de rappeler, cependant, que dans les théories traditionnelles et les débats sur les jeux (games) - y compris ceux à partir desquels une grande partie de l'étude des jeux numériques réalisée à ce jour a tiré la conceptualisation de nouvelles formes de «jeu numérique (digital play)»- la jouabilité est largement considérée comme individuelle, ou limitée à de petits groupes, et plutôt marginale à l'ordre social. Pour Caillois (2001) et Huizinga (1955), la plus grande signification sociale des jeux (games) se trouve dans les homologies entre leurs structures et les formes sociales; par exemple, entre les jeux de hasard (games of chance) et le marché boursier, ou les jeux d'adresse (games of skill) et les parcours de carrière. Pour Geertz (1973) et Sutton-Smith (1997), le jeu en groupe (group play) fournit une occasion importante, bien que surtout symbolique, de réordonner, de transgresser, et par ailleurs de donner du sens à des systèmes plus importants de l'ordre social (y compris les relations de pouvoir, les hiérarchies sociales, etc.). Ce qui se passe aujourd'hui, en revanche, est assez différent.

Comme décrit dans la partie précédente, ce n'est pas que l'ordre social récapitule certains éléments de jeux (games), mais plutôt que les jeux (games) eux-mêmes sont devenus des formes d'ordre social. Comme les jeux (games) deviennent rationalisés, les fonctions rationnelles fondamentales à tous les jeux formalisés (formal games) prennent une importance sans précédent. Finalement, ces jeux (games) commencent à produire leur propre forme de rationalité sociale, en imposant les trois types de pratiques rationnelles sur des millions de joueurs. De ce point de vue, il devient clair que l'institutionnalisation à multiples facettes des jeux dans les nouveaux processus de rationalisation sociale est la clé des changements dialectiques du jeu (play).

Pour expliquer cet état de choses, nous proposons que la jouabilité soit comprise comme un continuum dans lequel le joueur passe d'une humeur joueuse, d'une humeur ludique générale à l'état spécialisé de concentration requis pour la pratique de jeux (games) spécifiques puis, finalement, à l'orchestration centralisée de ce passage sur une échelle de masse autour des règles et des systèmes techniquement institués caractéristiques des jeux rationalisés (rationalized games). Dans cette dernière aptitude, la théorie doit tenir compte de la rationalisation des opérations de base de ces jeux, des relations de pouvoir et des conditions socio-culturelles qui définissent leurs règles et leurs paramètres, ainsi que des pratiques de jeu (play) émergentes et subversives qui en découlent. Nos points de départ pour l'élaboration de cette théorie sont la théorie réflexive du jeu de Bateson et la théorie du double aspect de la relation entre le jeu (play) et les jeux (games) de Walther (2003) 
Bateson affirme que «Le jeu (play) est paradoxal car il est à la fois dans et hors de notre espace social sémantique "normal" » (Walther, 2003). Du point de vue quotidien, normal, le jeu (play) a cette qualité paradoxale dans la mesure où il construit des structures imaginatives à partir de choses et de situations ordinaires, et introduit délibérément de l'ambiguïté dans des actions ordinaires. Comme Bateson le décrit (1973), le jeu (play) est " une méta-communication qui se réfere exclusivement à soi, et non à une source externe ou à un récepteur. » Bateson donne l'exemple des animaux faisant semblant de se battre. Ils doivent effectivement mordre l'autre, et pourtant le faire d'une certaine manière pour signifier que la morsure n'est pas un "vrai » mordre. Cette forme particulière de réflexivité est présente dans les activités quotidiennes ludiques de toutes sortes, et est sans doute la base psychique qui a organisé le jeu (play) et à partir de laquelle les jeux (games) sont construits. Dans ce sens, l'humeur ludique (playfulness) est une activité identifiable, mais elle ne relève pas d'un lieu précis. Il s'agit d'un type de jeu (play) localisé ou réactif, qui est contingent des structures et des thèmes stipulés par ce qui est alors interprété comme étant un non-jeu (non-play). Ainsi, dans le monde vécu, des moments indifférenciés d'humeur ludique adviennent aux côtés des autres pratiques communicatives de la vie quotidienne et les parasitent ; y compris bien sûr les activités "sérieuses", qui deviennent à leur tour définies comme telles que lorsqu'elles sont rapportées à l'humeur ludique. (Note 3)

Figure 1 - La rationalisation du jeu : une approche différenciée

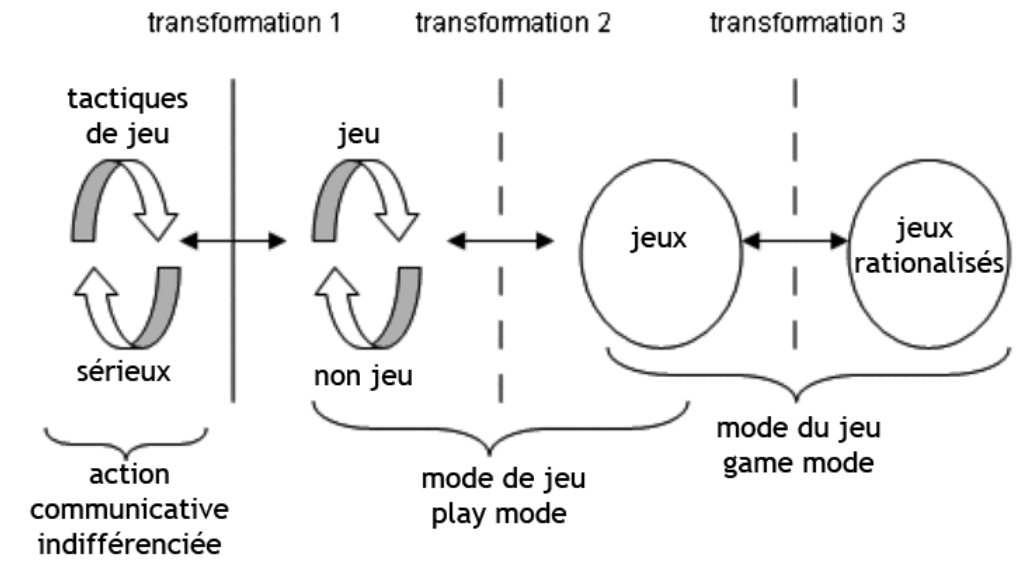

Walther emploie la théorie de la distinction de Spencer-Brown (1969), ainsi que la description du paradoxe du jeu (paradox of play) de Bateson (1973) pour identifier deux «transgressions» (nous préférons "transformations») qui permettent au joueur d'entrer dans l'état d'esprit requis pour s'engager réellement ou profondément dans le jeu (illustré comme les première et deuxième divisions dans la figure 1). La première représente le point où le joueur franchit la frontière qui sépare les pratiques communicatives indifférenciées de la vie quotidienne du domaine spécialisé du jeu (play). La seconde se produit lorsque le joueur se déplace d'un "état d'esprit ludique » général (general "play state ») vers l'état d'esprit plus concentré (the more focused game state $^{5}$ ) qui est requis pour participer efficacement à l'action d'un jeu en particulier, 
conformément à (ou à tout le moins avec une prise de conscience de) ses règles et critères spécifiques. Cette deuxième transformation est également en conformité avec la description de la transition de paidia vers ludus que propose Caillois (2001).

Walther (2003) décrit le point de vue de Spencer-Brown : « Un univers vient à être quand un espace est séparé, c'est à dire quand on fait une distinction. " Dans le jeu (play), cet " espace» commence comme une séparation purement métaphorique des sphères conçues par imagination, mais dans le cas des jeux (games), il se transforme en un lieu géographique réel. La «forme de la distinction » comprend à la fois l'espace différencié, qui devient le côté «marqué » de l'espace étant délimité par la distinction, ainsi que le reste, qui devient le côté «non marqué » de la distinction.

Premièrement, le jeu (play) devient le côté «marqué » de la distinction entre le jeu (play) et le monde vécu " ${ }^{6}$ lifeworld». Comme les joueurs entrent dans une humeur de ludisme, ils adoptent une perspective différenciée sur le jeu et non-jeu (play and nonplay). Pour Walther, c'est à ce stade que la réflexivité entre en jeu; mais nous considérons la réflexivité du ludisme comme une modification spécifique du type de réflexivité qui caractérise l'humeur ludique (playfulness) dans le monde vécu. La différence entre ce ludisme original (play mood) et l'humeur ludique est la tentative des acteurs pour donner une continuité dans le temps et l'espace à leur jeu (play) et au travail qu'ils accomplissent dans la construction d'un univers imaginaire. Une fois dans le domaine du jeu, toutes les activités qui n'entrent pas dans cet univers sont redéfinies comme « non-jeu » (non-play).

Pourtant, alors même que cette distinction initiale différencie certaines formes d'activité des pratiques communicatives indifférenciées du «non-jeu » (non-play), jouer, à ce stade, reste une notion très ouverte et changeante, qui se caractérise principalement par une limitation structurelle (boundedness) qui l'isole des structures, des préoccupations et des conséquences de la vie sociale " ordinaire ». C'est ce fait d'être limité qui permet au joueur de concentrer son attention sur (le jeu - play) les activités à portée de la main. Ceci évolue, cependant, lorsque le jeu (play) est canalisé dans des jeux (games), et qu'un système de règles est introduit. Walther (2003) décrit un jeu (game) comme une continuation de l'humeur ludique en ce qu'il adopte la pratique de la distinction qui est établie dans le jeu (play), "mais sa "loi" centrale est sa capacité unique à réduire la complexité du jeu via un ensemble d'objectifs bien définis, les règles non négociables. »Cette seconde transformation implique une augmentation de la rationalité au sens ordinaire du terme.

La figure 1 représente notre adaptation du modèle de Walther, y compris l'ajout de l'humeur ludique dans le monde vécu, à un extrême et, à l'autre, l'institution technologique des qualités rationnelles caractéristiques des jeux en ligne massivement multijoueurs et d'autres jeux rationalisés (rationalized games). Nous avons modifié le modèle de Walther pour illustrer le processus de rationalisation comme comprenant trois transformations. Alors que les conditions nécessaires pour que chacune de ces transformations se produise peuvent se manifester comme les caractéristiques des systèmes de jeu (game systems) ou des artefacts, elles doivent d'abord et avant tout être comprises comme des changements dans la relation entre le jeu (game) et ses joueurs. Les trois transformations doivent se produire pour qu'un jeu (game) commence à fonctionner comme un système de rationalité sociale. En référence au terme de Caillois pour le jeu rationnel (rational play) (ludus), ainsi que pour le domaine de la ludologie (ludology), nous appelons provisoirement ceci la théorie de la ludification. 
La première transformation (illustrée par la figure 1) a déjà été décrite comme le passage de l'humeur ludique quotidienne, à travers sa modification momentanée et non organisée, de contenu « sérieux » en mode de jeu (ludisme). Jouer, en ce sens, n'est pas encore contraint par des règles permanentes, et pas complètement séparé du monde du non-jeu (non-play) - la "réalité », qui, de temps en temps, menace d'intrusion. La deuxième transformation est longuement décrite dans la littérature sur le jeu (play). Ici, l'humeur ludique devient gouvernée par la règle, et l'ambiguïté du jeu libre est également réduite dans les contraintes des conditions temporelles et spatiales fixées par le jeu (game). Alors qu'ils sont encore caractérisés par l'humeur ludique, les jeux (games) sont aussi simultanément constitués par une ambiance de jeu (play mood) qui décrit un état de réflexivité accrue, impliquant les relations et les interactions du joueur avec les règles et les limites du jeu (game). Cela inclut le désir qu'a le joueur ainsi que les tentatives qu'il réalise en ce sens - pour gagner, pour découvrir la structure du jeu (game) et ses failles cachées, pour progresser ou avancer à travers les niveaux d'un jeu (game), ou pour établir des stratégies contre un concurrent. Jouer à un jeu (game) est donc un double processus, qui exige un équilibre délicat de jouer librement (playing) et suivre les règles (gaming). Comme Walther l'explique : « On doit se tenir à la distinction initiale (autrement on est avalé par l'autre du jeu), et il faut constamment accepter l'organisation, la structure générale du jeu » (2003).

Lorsque les jeux (games) sont techniquement médiés et commercialisés à grande échelle, comme dans l'exemple des jeux en ligne massivement multijoueurs, ou des sports professionnels, ils subissent une troisième transformation en jeux rationalisés (rationalized games). À ce stade, les propriétés rationnelles de la réflexivité, de la limitation, et de la réglementation, qui se retrouvent dans tous les jeux organisés (organized games), sont intensifiées d'une façon sans précédent. Cette intensification au travers de la médiation technique apporte de nouvelles qualités de précision au jeu (game). Les règles et les paramètres du système de jeu (game system) sont programmés dans le code du jeu (game code) et deviennent de plus en plus rigoureusement appliqués et optimisés. Le jeu (game) lui-même devient l'objet de formes de plus en plus précises de mesure et de calcul.

Même à ce stade, les joueurs possèdent une «initiative" (Feenberg, 1999), qui fait surface de diverses façons lorsqu'ils s'approprient la technologie. Les exemples les plus évidents sont le piratage (Kirkpatrick, 2004) et le modding (Postigo, 2003; Kücklich, 2005), mais l'initiative du joueur peut aussi se manifester de façon plus subtile. Il s'agit notamment des marchés non autorisés des items qui ont surgi autour de jeux (games) comme Everquest et World of Warcraft (Castronova, 2005), la collaboration pour le jeu de rôle (role-playing) et pour l'élaboration de communauté qui se produit sur certains serveurs ou dans certains groupes de joueurs (Taylor, 2006), la créativité et le bricolage culturel du joueur (Poremba, 2003), et l'échange de codes de jeu (game codes) et de « soluces » sur Internet (Consalvo, 2007). C'est ici que l'on découvre les vestiges du jeu non rationalisé, opérant tant à l'intérieur qu'en dehors des structures du jeu formalisé, occupant la "marge de manœuvre » qui coexiste avec le système enrégimenté du jeu rationalisé (rationalized play) (Feenberg, 1999).

Une grande partie de cette activité peut être décrite comme ludique, dans le sens que nous donnons à ce terme. Bien que le joueur conserve l'humeur ludique nécessaire pour supporter la condition expérientielle de jouer à un jeu (playing a game), les structures excessivement rigides des jeux rationalisés (rationalized dames) invitent une réponse 
ludique caractéristique des pratiques communicatives non différenciées du monde vécu non marqué (unmarked lifeworld). Dans ce contexte, comme Sutton-Smith le décrit, le ludisme peut être compris comme une sorte de "méta jeu» (metaplay) qui se trouve dans les activités et dans les attitudes "qui jouent avec les attentes normales du jeu (play) lui-même ", telles que "l'inversion, l'exagération, jouer avec les limites, [et] jouer avec l'espace et le temps » (1997, p. 147). C'est grâce à l'imprévu, ou aux activités «émergentes » des joueurs provenant du ludisme que le " potentiel latent technique » (Feenberg, 1999, p. 97) des technologies de jeux (games) numériques est peu à peu découvert.

Revenons à la figure 1. Nous proposons donc que, comme le concept de jeu (game) évolue, vers la droite, par l'intensification des principes de la rationalité sociale, il développe des propriétés qui permettent finalement sa transformation en un système de rationalité sociale. Le processus peut également fonctionner en sens inverse, lorsque l'on passe à un niveau moins important de rationalisation, conformément à une diminution de la présence ou de l'intensité des propriétés des jeux rationalisés (rationalized games). Nous avons provisoirement identifié ces propriétés comme la réflexivité, la limitation, la règlementation, la précision et le ludisme (tableau 1). En identifiant ces propriétés, nous ne tentons pas de définir le jeu (play) ou de décrire les jeux (games) de manière exhaustive. Nous proposons plutôt ces propriétés comme des caractéristiques clés du processus de ludification par lequel un jeu rationalisé (rationalized game) promulgue une forme d'ordre social.

Tableau 1 - Les cinq propriétés de la ludification

\begin{tabular}{|l|l|}
\hline Réflexivité & $\begin{array}{l}\text { À mesure que le jeu (play) devient rationalisé, il devient de plus en plus auto- } \\
\text { référentiel et exclu les thèmes et les activités extérieurs à la réalité construite de } \\
\text { la pratique du jeu (play activity). ou du jeu formalisé. Le système et les structures } \\
\text { du jeu (game), ainsi que le rôle du joueur, gagnent peu à peu la primauté aux } \\
\text { dépens d'un monde « extérieur » ou « réel » de plus en plus différencié. }\end{array}$ \\
\hline Limitation & $\begin{array}{l}\text { Puisque le jeu (play) est une activité différenciée, un niveau de limitation doit } \\
\text { toujours exister afin de distinguer le jeu (play) des pratiques communicatives } \\
\text { indifférenciées du monde vécu. Lorsque les jeux (games) deviennent rationalisés, } \\
\text { cependant, les limites - en termes de capacité, d'espace, et de possibilités pour le } \\
\text { jeu-deviennent plus limitatives, mieux définies, et indépendantes. }\end{array}$ \\
\hline Règlementation & $\begin{array}{l}\text { Quand le jeu (play) se transforme en un jeu (game), il devient assujetti à un } \\
\text { ensemble précis de règles et de paramètres. Quand les jeux (games) deviennent } \\
\text { rationalisés, leurs systèmes de règles deviennent plus rigides et exhaustifs parce } \\
\text { qu'ils sont déterminés au niveau technique et institutionnel. }\end{array}$ \\
\hline Précision & $\begin{array}{l}\text { La spécification et la normalisation des règles d'un jeu (game) sont accompagnées } \\
\text { d'une augmentation de la précision, ce qui permet la mesure et l'optimisation de } \\
\text { la jouabilité, à la fois en termes d'efforts et de résultats. Comme les règles, la } \\
\text { précision conduit à une réduction de la gamme de ce qui est possible dans un jeu } \\
\text { (game), et transforme le jeu (play) en un ensemble quantifiable et prévisible } \\
\text { d'activités. }\end{array}$ \\
\hline
\end{tabular}




\begin{tabular}{|l|l|}
\hline & $\begin{array}{l}\text { L'humeur ludique décrit la forme indifférenciée de jeu (play) qui se produit dans } \\
\text { les pratiques communicatives quotidiennes. Contrairement à la liberté } \\
\text { d'imagination propre au jeu (play), l'humeur ludique se caractérise par sa } \\
\text { ludique }\end{array}$ \\
& $\begin{array}{l}\text { situation interne et sa dépendance au système du jeu (game), grâce auxquelles il } \\
\text { en oriente les thèmes et le contenu. L'humeur ludique peut être subversif ou } \\
\text { réactif, mais il fonctionne toujours en interaction directe avec les règles, la } \\
\text { temporalité, la séquence, et les structures d'un jeu (game). }\end{array}$ \\
\hline
\end{tabular}

Alors que ces cinq propriétés doivent toutes être présentes pour qu'un jeu (game) fonctionne comme un système de rationalité sociale, chacune d'entre elles peut être établie structurellement (par exemple par les conventions, les normes, les conditions des contrats d'utilisation, etc.) ou technologiquement, dans la conception du système du jeu (game). La section suivante présente une étude de cas intégrant à la fois la ludification (tableau 1) et la rationalisation du jeu (play) (figure 1), à partir d'exemples tirés de World of Warcraft (WoW), ceci afin d'illustrer comment nous pourrions commencer à comprendre la ludification comme un processus qui favorise et active de nouvelles formes d'ordre social, aussi bien qu'il crée de nouvelles possibilités de résistance et d'innovation de la part des usagers dans les jeux en ligne massivement multijoueurs.

\section{3. Étude de cas : la ludification dans World of Wracraft (WoW)}

Lancé aux États-Unis en 2004, WoW reste l'un des jeux en ligne massivement multijoueurs les plus populaires de l'histoire du genre. Constamment classé dans les listes de best-seller, et souvent crédité pour avoir fait des jeux en ligne massivement multijoueurs une tendance dominante, WoW continue d'attirer l'attention du public. Le jeu (game) revendique actuellement une population de plus de 10 millions de joueurs à travers le monde (Blizzard Entertainment, Inc, 2008), générant des revenus annuels estimés à des centaines de millions (Vella, 2008). Parmi les études sur les jeux numériques, l'intérêt académique porté à WoW est donc conséquemment assez élevé, et au cours des deux dernières années, un grand nombre de recherches concernant les jeux en ligne massivement multijoueurs ont mis l'accent sur ce jeu (game) et sa population, son design, et son impact culturel. Cette recherche a produit de nombreux articles (par exemple, Williams et al., 2007 ; Bessière et al., 2007 ; Humphreys, 2008), un numéro spécial du journal Jeux et Culture (Krzywinska et Lowood, 2006), et au moins une collection éditée (Corneliussen et Rettberg, 2008).

Si beaucoup de choses ont maintenant été écrites au sujet des joueurs de WoW - sur leurs pratiques culturelles, les communautés, les interactions sociales et les comportements de jeu (in-game behaviors) - une attention moindre a été accordée aux structures technologiques, sociales et politiques sous-jacentes au jeu (game). Des études encore plus récentes sur les jeux en ligne massivement multijoueurs, et sur WoW en particulier, indiquent qu'il y a un besoin évident de poursuivre la recherche dans ce domaine. Comme Taylor (2006b, p. 319) l'écrit: «Plutôt que d'identifier simplement la "culture émergente" comme une propriété de premier ordre de la vie des jeux en ligne massivement multijoueurs et d'arrêter là, nous avons également besoin d'une meilleure 
compréhension de la nature complexe de la culture générée par le joueur (player-produced culture) et de sa relation aux artefacts techniques du jeu (game)", ainsi que de "comprendre le rôle que les systèmes de stratification et les formes de contrôle social jouent dans ces mondes de jeu (game worlds). » Ainsi, alors que notre utilisation de WoW comme cas d'étude se fonde sur un corpus représentant une part de la recherche relativement large, notre objectif de démontrer le rôle du jeu (game) comme système de rationalité sociale représente un écart important.

\section{1. Réflexivité}

Comme d'autres jeux numériques (digital game), WoW affiche et invite un haut niveau de réflexivité à travers la nature même de sa conception interactive. Comme le note Kirkpatrick, "Dans les jeux informatiques (computer games), l'engagement critique avec l'interface et l'ordinateur - en tant qu'il est une machine avec des règles de comportement compréhensibles, techniques -, est la norme. [...] Les jeux (games) utilisent les connaissances techniques et la compréhension du comportement de l'ordinateur pour savoir quand une solution appliquée à un jeu (game) fonctionnera probablement pour les autres » (2008, p. 128) quel que soit le contexte esthétique et narratif spécifique du jeu (game). Afin de participer à WoW, le joueur doit apprendre à manœuvrer dans l'environnement du jeu, il doit découvrir les clés de contrôle du jeu (game) (les touches à presser, et quand le faire), développer un certain sens de la mécanique du jeu (game) et de la gamme des actions possibles (au moins à un niveau d'introduction), et comprendre le système de niveaux et les crédits (système d'échange monétaire) propres au jeu (game). À mesure que les structures de base du jeu (game) se révèlent, l'engagement réflexif des joueurs devient de plus en plus sophistiqué, impliquant des activités telles qu'affiner les réglages de certaines compétences plutôt que d'autres afin de construire un personnage spécifique, ou réorganiser la configuration des touches du clavier pour augmenter la jouabilité.

La réflexivité s'accroît quand le joueur éprouve de la tension vis-à-vis des règles et des caractéristiques techniques du jeu (game). Pour exemple, les premières phases du jeu (game), quand un joueur apprend d'abord les règles, ou quand les joueurs sont incapables d'effectuer une action souhaitée (par exemple pour tenter de gravir une falaise infranchissable), ou lorsque le nombre de connexion est si élevé que cela force les joueurs à attendre avant de pouvoir se connecter à un serveur. En l'absence de ces tensions, les qualités restrictives et rationalisantes de la conception du jeu (game) sont principalement perçues comme des retours (feedback) dans un cycle d'interactivité, un peu comme les interactions, dans le monde "réel ", sont à la fois vécues comme à la fois limitées par et permises grâce aux lois physiques. Comme Rehak le fait valoir, ces interactions sont elles-mêmes un aspect agréable des jeux numériques (digital games), puisque "une partie de ce que les utilisateurs attendent des ordinateurs est la réponse continuelle à leurs propres actions - un reflet de leur capacité d'agir personnelle rendue disponible à l'écran pour un excédent de plaisir » (2003, p. 111 ).

Les points et les systèmes de niveaux attribués aux actions des joueurs, les items du jeu (game), étendent aussi la réflexivité en attirant l'attention du joueur sur les structures numériques sous-jacentes du jeu (game). Comme d'autres jeux numériques (digital games), WoW possède un système de niveaux prédéterminé et clairement indiqué, qui évalue quantitativement les actions des joueurs et les tâches qu'ils accomplissent 
(comme mener à bien des " quêtes 7 ", ouvrir une " instance de donjon ${ }^{8}$ ", ou vaincre vos adversaires lors d'un combat "joueur-contre-joueur » [PvP pour Player versus Player]) en leur attribuant une valeur exprimée en "points d'expérience ${ }^{9}$ " (XP). Tous les joueurs commencent au niveau 1 (sauf s'ils ont acheté un personnage déjà monté de niveau) et doivent accumuler une quantité suffisante de points d'expérience avant de passer au niveau suivant (compléter chaque niveau nécessitant toujours davantage de $\mathrm{XP}$ ) ce système est reproduit jusqu'à ce que le joueur atteigne le niveau 70 (le niveau maximal accessible au moment de notre étude, récemment porté à 80 ). En outre, les attributs spécifiques de chaque personnage, comme la force, l'endurance et l'intelligence, sont exprimés en chiffres, comme le sont la santé et le mana (l'énergie magique utilisée pour lancer des sorts), qui nécessitent un réapprovisionnement constant. D'un autre côté, la majorité des items du jeu (game), même les objets de quêtes, ont une valeur d'échange. Des items (et même des personnages complets) peuvent être achetés et vendus contre de l'Or (la monnaie utilisée dans WoW) ou échangés selon divers moyens, tant à travers le système de jeu (game) que par le commerce non autorisé sur le marché du « monde réel »(Castronova, 2005).

Les systèmes numériques du jeu (game) communiquent en permanence avec le joueur, dit Stallabrass, décrivant une idée de progrès sans ambiguïté qui est cette «toujours présente dans le jeu (game), poursuivant et interprétant l'action» (1996, p. 90). Alors que les joueurs sont toujours libres d'ignorer les structures numériques du jeu (game), il y a de nombreuses récompenses et de nombreux avantages associés au fait de «monter de niveau " (leveling up). Pour chaque nouveau niveau atteint, le joueur gagne également l'accès à de nouvelles quêtes (de plus en plus complexes, et aux enjeux de plus en plus élevés), de nouveaux items, de nouvelles compétences, et de nouveaux secteurs dans l'univers du jeu (game). La grande visibilité du système des points d'expérience, et le fait de privilégier les progrès enregistrés dans WoW, offrent aux joueurs un modèle clairement articulé de ce qu'est la "bonne » façon de jouer (même si elle n'est pas obligatoire) ; une façon de jouer qui révèle et met en évidence les critères de mesure même sur lesquels les actions du joueur sont évaluées.

\section{2. Limitation}

47 Malgré le fait que l'environnement de jeu (game environment) de WoW soit expansif, collaboratif, ouvert (open-ended), et en constante évolution, il est néanmoins limité par sa conception et son code de programmation. Le code du jeu (game code) définit la capacité et les limites de la grande majorité des activités du jeu - il constitue l'environnement virtuel, lui fournit un système de lois physiques, détermine l'éventail d'actions qui sont possibles (marcher, courir, s'asseoir, attaquer, jeter un sort), pour qui ces actions sont possibles (par exemple, seuls les paladins peuvent utiliser un sort de "bouclier divin»), et à quelle fréquence (par exemple, les pierres de foyer qui téléportent le joueur dans un "refuge " présélectionné ne peuvent pas être utilisées plus d'une fois par heure). Dans l'environnement de WoW, la capacité de ce qui est et n'est pas possible - en termes d'actions des joueurs et d'interactions avec l'environnement virtuel - n'est pas seulement découverte dans l'acte de jouer (comme dans les jeux non rationalisés), mais est techniquement mise en application par le moteur du jeu (game engine). Lorsqu'ils évoluent dans un jeu numérique, les joueurs interagissent avec la base de données via un analyseur syntaxique, qui lit les actions des joueurs comme une série de commandes « if-then $»^{10}$ (Kirkpatrick, 2004). Au niveau de 
l'interaction homme-ordinateur, la jouabilité est donc réductible à une série de variables, et les sélections sont toutes tirées d'un nombre immense, mais tout de même fini, de solutions possibles, exprimées dans le langage rudimentaire du code informatique.

Les déplacements et les choix qui n'ont pas été encodés dans le programme du jeu (game) ou que la conception de celui-ci ne permet pas (que ce soit intentionnellement ou non), sont tout simplement impossibles, à moins d'avoir recours à des interactions techniquement spécialisées comme le piratage informatique ou le modding. Dans le cas de WoW, qui a été spécifiquement conçu pour permettre au joueur de hauts niveaux de capacité d'agir et d'indépendance, la conception du jeu (game) permet même dans une certaine mesure une intervention technique. Comme Taylor le décrit, le système de jeu (game system) de WoW a été construit avec une interface utilisateur flexible, destinée à permettre aux « joueurs-développeurs » de pratiquer des modifications qui «ne sont pas simplement esthétiques, mais peuvent procurer une fonctionnalité fondamentale au jeu (game), voir changer la nature du jeu (play) lui-même " (2006, p. 326). En tout cas, puisque la majorité des joueurs n'ont pas les compétences techniques nécessaires pour intervenir à ce niveau, la plupart des actions des joueurs demeurent strictement dans le champ d'application de ce qui est prévu par le moteur de jeu Blizzard ${ }^{11}$.

Cela ne signifie pas que tous les mouvements possibles ou tous les résultats ont été imaginés ou prédéterminés par les concepteurs du jeu (game). Les joueurs se livrent à une variété de comportements imprévus et même non autorisés, de tricheries et de " gold-farming ${ }^{12}$ » pour acheter et vendre des personnages sur le marché du monde réel (Castronova, 2005). Les joueurs affectent l'environnement du jeu (game environment) à des fins sociales et créatives variées, cela allant du fait d'initier et entretenir des relations personnelles à l'utilisation du jeu (game) comme une base pour la production de « Machinima $»^{13}$ (Lowood, 2006) (Note 4). Des recherches antérieures ont également identifié de nombreux exemples de «jeux émergents (emergent play)» dans WoW, dont un certain nombre d'incidents impliquant de grand nombres de joueurs qui organisaient une manifestation collaborative en se réunissant à un moment précis et dans un lieu spécifique, dans le but de surcharger (et donc de bloquer) un serveur, ce qui était, pour eux, un moyen de communiquer leur revendication à Blizzard et aux autres joueurs (Taylor, 2006c).

Le jeu (game) contient aussi des ratés occasionnels, et il produit des résultats imprévus, ce qui lui ajoute des qualités « émergentes ». En 2007, par exemple, WoW été frappé par " une pandémie » non planifiée, qui a émergé inopinément à partir d'un sort destiné à la propagation d'une maladie infectieuse parmi un groupe limité de joueurs de niveau avancé, dans le contexte spécifique d'un cas de "boss battle » de donjon (le boss est le dernier monstre d'un niveau ou d'une quête, généralement de loin le plus difficile) (Balicer, 2007 ; Lofgren et Fefferman, 2007). Plus de quatre millions de joueurs ont été infectés au cours de la pandémie dite du "Sang Corrompu », provoquant le genre de " chaos social qui vient d'une épidémie à grande échelle déclenchée par une maladie mortelle " (Lofgren et Fefferman, 2007, p. 625). Il est important de se rappeler, toutefois, que ces types d'événements se produisent néanmoins dans un domaine préétabli de possibilités, délimité par les affordances technologiques du jeu (game) - même si certains de ces affordances peuvent ne pas avoir encore été découvertes ni par les joueurs, ni par les concepteurs du jeu (game), avant qu'elles n'éclatent. 
51 S'il y a une autre façon dont WoW expose des caractéristiques de limitation, c'est bien à travers son récit et ses particularités esthétiques. Grâce à la combinaison de graphismes riches, d'une architecture sonore, et d'une spatialité, WoW offre aux joueurs un « monde » de jeu (game) extrêmement détaillé et cohérent. À mesure que les techniques d'animation par ordinateur en trois dimensions (3D), les technologies de modélisation et l'ingénierie du son dans les jeux numériques deviennent plus sophistiquées et complexes, les environnements spatiaux et artificiels du jeu (game) sont non seulement de plus en plus prédéterminés, mais aussi de plus en plus immersifs, construisant un espace de jeu (play space) nettement délimité, dont les limites sont renforcées par la logique interne du jeu (game). Les affordances et les limites du code source ne sont donc pas simplement perçues comme établissant la jouabilité autorisée, mais aussi comme constituant la réalité « physique » du monde du jeu (game world).

52 La naturalisation de la conception du jeu (game) et des paramètres est facilitée par l' « interface utilisateur graphique » (GUI), ce qui empêche la plupart des joueurs de s'engager directement avec le potentiel infini du «jeu en tant que code " (Kirkpatrick, 2004). Le joueur est isolé du code, qui est l'objet sous-jacent de ses actions. Le système de contrôle, ou "l'interface entre le joueur et le système d'exploitation » (Stallabrass, 1996, p. 96), traduit les actions souhaitées par joueur à l'analyseur «derrière l'écran ». À mesure que le joueur apprend la conception et les paramètres du code source, ils deviennent intériorisés comme faisant partie de la réalité « physique » du monde du jeu (game world). Ces paramètres, en liaison avec les normes et conventions créés par la communauté des joueurs, en viennent à définir ce que le jeu (game) est, ainsi que ce qu'il n'est pas.

\section{3. Règlementation}

Ces deux premières propriétés de la ludification (réflexivité et limitation) sont étroitement liées à la troisième propriété, la règlementation. Comme décrit précédemment, à la différence des systèmes de règles des jeux non rationalisés (nonrationalized games), les règles médiées technologiquement sont rigides et précises, et ne peuvent pas être négociées ou contestées par le joueur moyen type, non spécialiste. Dans WoW, la plupart des règles du jeu (game) et des paramètres sont établis, maintenus et communiqués par la base de données du jeu (game), et donc intégrés dans la conception technologique du jeu (game) lui-même. Les «lois» de ce système peuvent donc être appliquées de manière parfaitement explicite, et intégrées dans la structure même de l'espace du jeu (game) (y compris dans ses dimensions esthétiques, spatiales, et dans son environnement), ainsi que dans la conception du jeu (game). À l'intérieur de WoW, cependant, la médiation technologique n'est qu'une des façons dont les règles viennent structurer la jouabilité et le comportement des joueurs ; cela fonctionne aussi en conjonction avec les systèmes formels et informels de surveillance, les droits des sociétés, les normes du groupe et les attentes des joueurs.

Comme il est pratique courante chez les opérateurs commerciaux des jeux en ligne massivement multijoueurs, Blizzard demande aux joueurs de WoW d'agréer à un contrat de licence finale d'utilisation (CLUF : Contrat de licence utilisateur final) et à un autre relatif aux conditions d'utilisation (TOS : Terms Of Service) avant d'entrer dans le jeu (game). Les activités des joueurs et les communications durant le jeu (game) sont 
ensuite contrôlées, à la fois par des systèmes automatisés inhérents au jeu (game) et par les employés de Blizzard, pour assurer continuellement une conformité. En plus de faire de la conformité aux "règles de conduite» formalisées du jeu (game) une condition d'utilisation (ce qui signifie que le compte d'un joueur peut être gelé ou supprimé si il désobéit), ces contrats demandent que les joueurs renoncent à un certain nombre de leurs droits tant qu'ils se trouvent dans l'environnement du jeu (game), y compris «le droit de posséder les fruits de son travail, les droits de réunion, les droits à la liberté d'expression" (Castronova, 2003, p. 8). De cette façon, soutiennent Herman, Coombe et Kaye (2006, p.191), WoW établit ses propres "formes de gouvernance et d'économie morale de la pratique » à laquelle les joueurs doivent se soumettre, ou sinon ils risquent l'expulsion. En outre, bon nombre des conditions énoncées dans le CLUF et le TOS cherchent à engager les joueurs dans des relations juridiques qui vont bien au-delà des limites du jeu (game). Un exemple clé de ceci : la clause abusive sur la propriété intellectuelle incluse dans le CLUF, qui revendique les droits de propriété exclusifs sur tout ce que les joueurs disent ou font tant qu'ils sont dans l'environnement du jeu (game).

À l'intérieur de WoW, les règles sont également institutionnalisées au niveau social par les normes et les attentes des communautés. Une grande partie de ce qui rend le fait de jouer à un jeu (game) en ligne agréable est sa capacité à proposer une dynamique sociale relativement développée, et une partie de ceci implique la construction et la négociation de normes sociales. Certaines règles informelles de jeu (informal rules of play) proviennent de la narration du jeu (game) et des conventions du genre (par exemple, chaque personnage est membre soit de l'Alliance, soit de la Horde, donc chacun vient avec sa propre histoire et ses attentes particulières), tandis que d'autres pourraient résulter $\mathrm{du}$ « code de conduite » d'une guilde particulièrement populaire ou très en vue (guilde : groupe de joueurs formalisé dans la conception du jeu). Certains ressortent du consensus de la grande communauté des joueurs, tandis que d'autres représentent les perspectives et les interprétations d'un petit nombre de joueurs particulièrement revendicatifs. À certains moments, les normes de la communauté viennent à fonctionner comme des systèmes de contrôle social qui œuvrent pour discipliner, pour exclure ou encore classer les joueurs et les comportements. Par exemple, dans son étude ethnographique récente à propos de WoW, Taylor (2006b) a découvert de nombreux exemples de guildes exigeant un âge minimum, et excluant formellement les joueurs âgés de moins de 18 ans.

\section{4. Précision}

Comme décrit précédemment, dans WoW, le jeu (game play) est optimisé et calculé au moyen d'un système de niveaux et d'économies virtuelles basés sur le capitalisme, qui servent à mesurer les activités du joueur et à évaluer ses actions et ses progrès. D'une certaine façon, les systèmes de niveaux de WoW s'appuient sur les conventions établies dans la tradition des jeux de rôle sur table (tels que "Donjons et Dragons»), qui utilisent un ensemble spécial de dés pour déterminer le résultat des événements et les actions $\mathrm{du}$ joueur. Toutefois, ces systèmes sont également des sous-produits de la numérisation, qui permet d'accéder à des niveaux jusque-là inimaginables de précision dans la mesure, l'enregistrement et l'analyse des activités en ligne de n'importe quel nombre de joueurs. La numérisation permet non seulement aux règles et aux structures du jeu (game) de devenir des réalités virtuelles immuables, mais elle transforme aussi 
les actions des joueurs, les communications durant le jeu (game), et les contributions créatives en données parfaitement standardisées et facilement récupérables (Mosco, 2004). Cela permet une surveillance continue et assez détaillée des activités et des interactions des joueurs.

Une connaissance précise des joueurs individuels facilite grandement la régulation du comportement des joueurs, ainsi que l'application des règles et des autres «conditions d'utilisation». Mais, plus important encore, une fois que les communications, les contributions et les activités des joueurs ont été numérisées et enregistrées, les données peuvent ensuite être triées, extraites, et être utilisées à / servir des fins commerciales variées. Selon Mosco, la numérisation «étend la marchandisation des contenus par l'extension des possibilités de mesurer et de surveiller, d'emballer et de reconditionner le divertissement et l'information» (2004, p. 156). Les concepteurs de jeux (games) utilisent des systèmes complexes de suivi et d'extraction des données pour découvrir les nouveaux modes de comportement et les préférences des joueurs, qui peuvent ensuite être utilisés pour améliorer ou élargir la conception du jeu (game) (par des patches ${ }^{14}$ ou des ensembles d'expansion). Ils peuvent aussi compiler les données sous diverses formes pour créer des rapports très détaillés sur les comportements de l'utilisateur, qui peuvent ensuite être vendus à des entreprises extérieures pour être utilisées dans des campagnes de publicité ou autres initiatives de marketing (Kline et al., 2003).

58 Le principe de précision se répand aussi bien dans les pratiques des joueurs. Non seulement les joueurs expérimentent la mesure précise de leurs pouvoirs et de leurs statuts par le jeu (game) tel que décrit précédemment, mais ils participent aussi à la mesure eux-mêmes. Un exemple récent, décrit par Taylor (2006b), est cette utilisation croissante d'interventions mods ${ }^{15}$ qui permettent une évaluation précise des actions des joueurs par d'autres joueurs. Ces mods, produites par les joueurs, non seulement facilitent une «focalisation grandissante sur la quantification» (p. 332) entre les joueurs qui les utilisent, mais permettent aussi aux joueurs de s'engager dans de nouvelles formes de coercition sociale, chacun évaluant la performance des autres à travers la lentille apparemment objective des outils de mesure. Comme l'écrit Taylor, "par leur rationalisation et la quantification de l'action, ils façonnent également fortement (et potentiellement ils limitent) ce qui est considéré comme un "bon jeu (good play)" ou ce qui est considéré comme raisonnable » (p. 332).

\section{5. L'humeur ludique}

La dernière propriété, l'humeur ludique, décrit la relation des joueurs à la rationalité sociale du jeu (game), et la façon dont ils la négocient. Les codes sources et les bases de données établissent quelles actions sont possibles dans l'environnement de jeu (game) de WoW, ce qui réduit considérablement les occasions de libre imagination. Dans le même temps, les propriétés réflexives du jeu (game) invitent le lecteur à s'engager dans des formes d'activités auto-référentielles, par exemple découvrir les limites et les affordances de la conception du jeu (game). Parce que l'humeur ludique consiste en une approche du jeu qui est réactive et structurellement incorporée au jeu (play), elle se manifeste et se produit dans le dialogue avec les structures sous-jacentes du jeu, jouant avec et occasionnellement contre le système. Ce changement dans l'orientation et le contenu des activités des joueurs est un facteur clé dans la part d'imprévu de la 
jouabilité (y compris la dotation des joueurs, la subversion, et l'innovation) qui continue à se déployer même au sein des jeux (games) hautement structurés et rationalisés. L'humeur ludique apporte un niveau plus élevé d'initiative vis-à-vis du système du jeu numérique (digital game).

Grâce à l'humeur ludique, le joueur participe, subvertit, et réinterprète les règles et les lois imposées par le système technique. Dans chacune des sections précédentes (réflexivité, limitation, réglementation et précision), un grand nombre des pratiques du joueur que nous avons décrites sont aussi des exemples de l'humeur ludique. Elles vont des explorations empiriques basiques de la mécanique du jeu (game) aux actions transgressives des joueurs qui ont aggravé la pandémie «Sang Corrompu » en diffusant délibérément l'infection, jusqu'au développement de mods qui mettent à découvert la logique numérique sous-jacente des actions du joueur.

61 Le potentiel subversif de l'humeur ludique est évident dans le piratage et le modding, mais il affleure aussi dans les pratiques quotidiennes du joueur, de l'élaboration concertée de normes sociales à la pratique de coordination entraînant le plantage du serveur en tant que forme de protestation. L'humeur ludique peut contribuer à la conception technologique des jeux numériques (digital games) de manière imprévue. Bien sûr, l'initiative du joueur peut aussi rencontrer des résistances - venant d'autres joueurs, si l'activité interfère avec leur propre jeu (play), ou des concepteurs du jeu (game), si l'activité interfère avec les objectifs de conception ou les priorités organisationnelles. Mais les initiatives inattendues et indirectes du joueur peuvent mettre à jour le «potentiel technique non réalisé » (Feenberg, 1999, p. 97) des technologies du jeu numérique (digital game). C'est ici que la rationalisation démocratique de cette forme technologique devient possible (Feenberg, 1999).

\section{Conclusion}

Bien que les caractéristiques politiques, culturelles, économiques et technologiques des jeux en ligne massivement multijoueurs soient toutes soumises à une attention constante et à une analyse dans les études sur les jeux (games), à ce jour, la littérature n'a jusqu'à présent pas réussi à relier de manière adéquate ces processus à la rationalisation généralisée du jeu (play), aux loisirs, et au monde vécu dans son ensemble. Nous avons cherché à remédier à cet oubli en posant les jeux (games) comme des systèmes de rationalité sociale opérant dans le contexte socio-historique plus large de la modernité, et en fournissant une structure (la ludification) pour une exploration plus complète des processus au travers desquels les règles du jeu (game rules) deviennent médiées techniquement, les pratiques de jeu s'institutionnalisent, et les joueurs deviennent rationalisés (et professionnalisés ou marchandisés). En outre, une compréhension plus complète des changements contemporains quant au rôle et à la fonction du jeu (play) en ce qu'il devient un processus de rationalisation de la modernité constitue un point d'entrée unique pour les discussions sur la marchandisation et la médiation technique des loisirs, un point d'entrée qui transcende la relation binaire travail/jeu, cette relation obsolète qui informe pourtant une grande partie de la littérature à ce jour.

63 En proposant que les jeux (games) puissent fonctionner comme des systèmes de rationalité sociale, nous avons tenté de construire une théorie du jeu qui prenne en compte la nature changeante des jeux (games) ainsi que leur fonction au sein des 
sociétés capitalistes contemporaines. Nous avons identifié cinq propriétés de la ludification, qui expliquent comment les jeux (games), découlant au départ de pratiques communicatives indifférenciées, évoluent graduellement vers une forme d'activité plus rationnelle (figure 1). La théorie de la ludification montre comment les propriétés essentielles des jeux (games) se prêtent à une appropriation et à une transformation dans les systèmes de la rationalité sociale. Notre théorie explique comment le jeu (play) vient à fonctionner comme une source de l'ordre institutionnel, en adoptant ces mêmes principes que l'on retrouve dans d'autres processus de rationalisation plus communément reconnus, des processus tels que la technologisation, la bureaucratisation et la marchandisation.

64 Comme cela a été vu dans le cas de WoW, la médiation technique ouvre les jeux (games) à d'autres processus de rationalisation, tels que la marchandisation. La congruence entre les divers systèmes rationalisés est un élément clé pour comprendre comment le jeu (play) s'inscrit dans le projet plus vaste de la modernité. Dans chaque cas, la technologisation du jeu (game) investit les propriétés identifiées dans la théorie de la ludification, avec cette nouvelle signification de structures de la rationalité sociale. Compte tenu des évolutions récentes dans le domaine du jeu en ligne massivement multijoueurs, comme les débats autour de la légalité des accords de licence (CLUF,) et ceux relatifs à la préoccupation croissante du public quant à la protection d'informations personnelles numérisées, il semble particulièrement opportun et nécessaire de mettre en place une approche qui considère la façon dont la rationalisation effective dans un secteur de la vie sociale conduit à une compatibilité accrue avec d'autres sphères rationalisées.

À cette fin, nous avons proposé de prendre pour base la théorie de la ludification pour mener une étude critique des formes de jeux rationalisés (rationalized play forms) - une étude qui comprend WoW, mais n'y est certainement pas limitée. Les futurs travaux dans ce domaine devraient se concentrer sur l'extension de son application non seulement à d'autres jeux en ligne massivement multijoueurs, mais aussi à d'autres formes de jeux multijoueurs techniquement médiés. Il est tout aussi important de continuer l'exploration des propriétés de l'humeur ludique, ainsi que les possibilités de rationalisation démocratique au sein de tous les systèmes de rationalité sociale (Feenberg, 2008). En fin de compte, l'étude des jeux (games) doit toujours être consciente du fait que le jeu numérique en ligne (online digital play) est bien plus qu'un divertissement technologique. Il génère aussi des communautés virtuelles dans lesquelles des systèmes rationnels de commerce, de technologie, et la jouabilité interagissent pour produire une expérience sociale plurielle.

\section{BIBLIOGRAPHY}

Balicer, R. D. (2007), “Modeling infectious diseases dissemination through online role-playing

games", Epidemiology 18(2): 260-261.

Bateson, G. (1973), Steps to an ecology of mind. New York: Ballantine Books. 
Bessière, K., Seay A. F., and Kiesler S. (2007) "The ideal elf: Identity exploration in World of Warcraft", CyberPsychology \& Behavior 10(4):530-535.

Blizzard Entertainment, Inc. (2008), World of Warcraft reaches new milestone: 10 Million subscribers. Press release, January 22, 2008. http://www.blizzard.com/us/press/080122.html (accessed September 5, 2008).

Bond. P. (2008), “Video game sales on winning streak, study projects”, Hollywood Reporter June 18.

Bourdieu, P. (1991), "Sport and social-class". In Rethinking popular culture: Contemporary perspectives in cultural studies, eds. C. Mukerji and M. Schudson, p. 357-373. Berkeley, CA: University of California Press.

Brookey, R. A., and Booth P. (2006), "Restricted play: Synergy and the limits of interactivity in The Lord of the Rings: The Return of the King video game", Games and Culture 1(3): 214-230.

Caillois, R. (2001), Man, play and games. Chicago: University of Illinois Press.

Castranova, E. (2003), “The right to play”, Paper presented at State of Play Conference, New York Law School, 13-14 November.

Castronova, E. (2005), Synthetic worlds: The business and culture of online games. Chicago: University of Chicago Press.

Coleman, S., and Dyer-Witheford N. (2007), "Playing on the digital commons: collectivities, capital and contestation in videogame culture", Media, Culture \& Society 29(6): 934-953.

Consalvo, M. (2007), Cheating: Gaining advantage in videogames. Cambridge, MA: MIT Press. Corneliussen, H. G., and Walker Rettberg J., eds. (2008), Digital culture, play, and identity: AWorld ofWarcraftreader. Cambridge, MA: MIT Press.

de Certeau, M. (1984), The practice of everyday life. Berkley/Los Angeles, CA: University of California Press.

de Peuter, G., and Dyer-Witheford N. (2005), “A playful multitude? Mobilising and countermobilising immaterial game labour”, Fibreculture 5.

Feenberg, A. (1992), “Subversive rationalization: Technology, power and democracy”, Inquiry September/December: 301-322.

Feenberg, A. (1995), Alternative modernity: The technical turn in philosophy and social theory. Berkley: University of California Press.

Feenberg, A. (1999), Questioning technology. New York: Routledge.

Feenberg, A. (2002), Transforming technology: A critical theory revisited. New York: Oxford University Press.

Feenberg, A. (2008), "From critical theory of technology to the rational critique of rationality", Social Epistemology 22(1):5-28.

Geertz, C. (1973), The interpretation of cultures. New York: Basic Books.

Grimes, S. M. (2006), “Online multiplayer games: A virtual space for intellectual property debates?”, New Media \& Society 8(6):969-990.

Grimes, S. M. (2008), “The exploitation of children's affective labour in corporately owned virtual worlds." Paper presented at the Joint Annual Meetings of Law and Society Association and Canadian Law and Society Association, Montreal, Quebec. May 29 - June 1.

Gruneau, R. (1999), Class, sports, and social development (2nd ed.). Champaign, IL: Human Kinetics. 
Habermas, J. (1984), The theory of communicative action: Lifeworld and system: A critique of functionalist reason (trans. T. McCarthy). Boston: Beacon Press.

Hans, J. S. (1981), The play of the world. Amherst, MA: University of Massachusetts Press.

Henricks, T. S. (2006), Play reconsidered: Sociological perspectives on human expression. Urbana/ Chicago, IL: University of Illinois Press.

Herman, A., Coombe R. J., and Kaye L. (2006), "Your second life? Goodwill and the performativity of intellectual property in online digital gaming”. Cultural Studies 20(2-3):184-210.

Huizinga, J. (1950/1955), Homo ludens: A study of the play element in culture. Boston: Beacon Press.

Hughes, L. (1995), “Children's games and gaming.” In Children's folklore: A source book, eds. B. Sutton-Smith, J. Mechling, T. W. Johnson, and F. R. McMahon, p. 93-119. New York: Garland. Humphreys, S. (2008), "Ruling the virtual world." European Journal of Cultural Studies 11(2):149-171. Juul, J. (2005), Half-real: Video games between real rules and fictional worlds. Cambridge, MA: MIT Press.

Kirkpatrick, G. (2004), Critical technology: A social theory of personal computing. Burlington, VT: Ashgate.

Kirkpatrick, G. (2007), "Between art and gameness: Critical theory and computer games aesthetics.” Thesis Eleven 89:74-93.

Kirkpatrick, G. (2008), Technology and social power. New York: Palgrave Macmillan.

Kline, S., de Peuter G., and Dyer-Witheford N. (2003), Digital play: The interaction of technology, culture, and marketing. Montreal, QC: McGill-Queen's University Press.

Krzywinska, T., and Lowood H. (2006), Guest editors' introduction. Games and Culture 1(4):279-280.

Kücklich, J. (2005), "Precarious playbour: Modders and the digital games industry." Fibreculture 5. http://journal.fibreculture.org/issue5/kucklich.html (accessed June 15, 2008).

Lasch, C. 1979. The culture of narcissism: American life in an age of diminishing expectations. New York: Norton.

Lofgren, E. T., and Fefferman N. H. (2007), "The untapped potential of virtual game worlds to shed light on real world epidemics." Lancet Infectious Diseases 7(9):625-629.

Lowenstein, D. (2005), Electronic Entertainment Expo (E3) 2005 state of the industry address. 11th Electronic Entertainment Expo (E3Expo), Los Angeles, CA. May 25.

Lowood, H. (2006), "Storyline, dance/music, or PvP? Game movies and community players in World of Warcraft." Games and Culture 1(4):362-382.

Marcuse, H. (1969), An essay on liberation. Boston: Beacon Press.

Mills, C. W. (1956), The power elite. New York: Oxford University Press.

Mosco, V. (2004), The digital sublime: Myth, power, and cyberspace. Cambridge, MA: MIT Press.

Pitts, R. (2006), Secret sauce: The rise of Blizzard. The Escapist June 6, issue 48. http:// www.escapistmagazine.com/issue/48/3 (accessed June 15, 2006).

Poremba, C. (2003), "Patches of peace: Tiny signs of agency in digital games." Level Up Conference Proceedings, University of Utrecht, The Netherlands, 4-6 November.

Postigo, J. (2003), "From Pong to Planet Quake: Post-industrial transitions from leisure to work", Information, Communication \& Society 6(4):593-607. 
Rehak, B. (2003), "Playing at being: Psychoanalysis and the avatar", In The video game theory reader, eds. M. Wolf and B. Perron, p. 103-127. New York: Routledge.

Rifkin, J. (2000), The age of access: The new culture of hypercapitalism where all of life is a paid-for experience. New York: Jeremy P. Thatcher/Putnam.

Salen, K., and Zimmerman E. (2003), Rules of play: Game design fundamentals. Cambridge, MA: MIT Press.

Schwartzman, H. B. (1978), Transformations: The anthropology of children's play. New York: Plenum Press.

Spencer-Brown, G. (1969), Laws of form. London: George Allen and Unwin.

Stallabrass, J. (1996), Gargantua: Manufactured mass culture. New York: Verso.

Stallybrass, P., and White A. (1986), The politics and poetics of transgression. London: Methuen.

Suits, B. H. (1978), The grasshopper: Games, life and Utopia. Toronto: University of Toronto Press.

Sutton-Smith, B. (1997), The ambiguity of play. Cambridge, MA: Harvard University Press.

Taylor, T. L. (2002), "Whose game is this anyway?: Negotiating corporate ownership in a virtual world", In Proceedings of computer games and digital cultures conference, ed. Mayra. Tampere, Finland: Tampere University Press.

Taylor, T. L. (2006a), Play between worlds: Exploring online game culture. Cambridge, MA: MIT Press.

Taylor, T. L. (2006b), “DoesWoW change everything?: How a PvP server, multinational player base, and surveillance mod scene caused me pause", Games and Culture 1(4):318-337.

Taylor, T. L. (2006c), "Beyond management: Considering participatory design and governance in player culture”, First Monday 7. http://firstmonday.org/issues/issue119/taylor/index.html (accessed September 11, 2008)

Thompson, P. (2006), "Commodification and secondary rationalization", In Democratizing technology: Andrew Feenberg's critical theory of technology, ed. T. J. Veak, p. 112-135. Albany, NY: SUNY Press.

Turner, V. (1974), Dramas, fields and metaphors: Symbolic action in human society. Ithaca, NY: Cornell University Press.

Vella, M. (2008), "Inside the world of Blizzard", Business Week Online August 20.

Walther, B. K. (2003), "Playing and gaming: reflections and classifications", Game Studies: The International Journal of Computer Game Research 3(1). http://www.gamestudies.org/0301/walther/ (accessed February 12, 2005).

Weber, M. (1958), The protestant ethics and the spirit of capitalism, trans. T. Parsons. New York: Scribners.

Williams, D., Caplan S., and Xiong L., (2007), "Can you hear me now? The impact of voice in an online gaming community", Human Communication Research 33(4):427-449.

\section{APPENDIXES}

\section{Notes de l'auteur}

1. Pour en savoir plus sur la théorie de la rationalité sociale, voir Feenberg (2008). 
2. Nous sommes ici inspirés par l'extension de la théorie de l'instrumentalisation de Feenberg à l'étude de la marchandisation, que Thompson a proposée (2006). Thompson fonde son remaniement de la notion d'instrumentalisation secondaire de Feenberg au sein des théories économiques et critiques, en découvrant les quatre caractéristiques ou les processus fondamentaux - à un système de marchandisation (l'aliénation, l'exclusion, la rivalité, et la normalisation).

3. Le concept de l'humeur ludique n'est pas sans rappeler la notion de « la perruque » proposée par de Certeau (1984, p. 29), dans laquelle les caractères de structure et de sérieux propres au travail, et la spontanéité ouverte propre aux loisirs, sont considérées comme « allant de paire », se répétant et se renforçant mutuellement. Bien que l'humeur ludique puisse fonctionner conformément à et au travers de la subversion des règles et des structures du système de recouvrement, il fonctionne toujours seulement dans une relation (parasite) au système.

4. «Machinima» se compose de films réalisés par des joueurs-producteurs, des films fabriqués à partir des jeux (games), soit par bricolage (le jeu est tout simplement enregistré et monté de façon à construire un texte nouveau ou un dérivé), soit en utilisant l'environnement du jeu (game) comme le plateau virtuel d'un film dans lequel les joueurs interprètent des scènes et des scripts.

\section{NOTES}

1. Le modding est la version modifiée d'un jeu où, par exemple, les personnages, les décors ou les cartes de jeux ont été modifiées par des joueurs. Ndt

2. Accessoires et objets utilisés par les avatars dans le jeu: armes, outils, vêtements, objets magiques, monnaie... Ndt

3. Les fanfictions sont des fictions écrites par un fan à partir des éléments du jeu (environnement, scénario, personnages, etc.). $\mathrm{Ndt}$

4. Les soluces sont des astuces, solutions et codes concernant le jeu qui sont délivrés par des joueurs pour aider les autres dans leur progression dans le jeu. Souvent, une soluce permet de court-circuiter le court normal du jeu ainsi que les règles. $\mathrm{Ndt}$

5. La différence entre play state et game state peut être indiquée ainsi : play state réfère à l'action de jouer, au concept de jeu en général, et conserve une tonalité ludique; alors que game state implique un jeu particulier, donc un système de règles, et insiste sur l'engagement du joueur quant au jeu - peut être une certaine idée d'esprit de compétitivité. Ndt

6. Monde vécu traduit ici lifeworld; impossible de dire si c'est ou non une référence à la position de Husserl selon laquelle la conscience constitue le sens du monde, que Feenberg cite dans d'autres textes. Ndt

7. Les quêtes forment la base des gains en expérience (points d'expérience : XP) dans le jeu World of Warcraft. Certaines s'accompagnent aussi de récompenses qui peuvent être rétribuées par le gain d'objets, ou dans la monnaie du jeu. $\mathrm{Ndt}$

8. «Instance » est un mot dont le sens a été directement dérivé de la signification qu'il a dans le langage informatique : une instance est la copie dans un espace privé d'une partie du programme général, chaque copie ayant un comportement indépendant des autres copies. Dans WoW, une zone instanciée est une copie conforme d'un donjon qui est réservée à un groupe de joueurs donné. Seuls votre personnage et les membres de votre groupe pourront y accéder. Leur équilibre et leur conception sont spécialement conçus pour le jeu en groupe. $\mathrm{Ndt}$ 
9. Les points d'expérience permettent au joueur de renforcer leurs compétences ou d'en acquérir de nouvelles, d'avoir accès à d'autres « lieux » du jeu, et de monter de niveau. $\mathrm{Ndt}$

10. If-then-else : instructions conditionnelles utilisées en programmation structurée. Ndt

11. Firme conceptrice et détentrice des droits de WoW. Ndt

12. Un gold farmer, littéralement un «fermier » qui « cultive » de l'or, est une personne répétant une série d'actions dans un jeu afin d'y acquérir des items (par exemple de la monnaie ou des objets virtuels). Le gold-farming est l'activité qui en découle. Par exemple : tuer sans cesse le même monstre grâce à une astuce permettant de le faire très rapidement permet de gagner des $\mathrm{XP}$ et des récompenses. Certains gold farmers revendent ensuite les récompenses (items ou monnaie virtuelle du jeu) dans le monde réel - cela s'appelle du real-money trading. $\mathrm{Ndt}$

13. Les machinimas sont un exemple de jouabilité émergente (emergent gameplay), terme regroupant toutes les utilisations nouvelles et inattendues d'un jeu vidéo. $\mathrm{Ndt}$

14. Le Patch désigne la mise à jour d'un jeu vidéo. C'est un fichier qui corrige les défauts du jeu. Ce terme peut également désigner un complément du jeu qui permet d'ajouter des fonctionnalités au jeu. $\mathrm{Ndt}$

15. Adjectif dérivé de modding. Ndt

\section{ABSTRACTS}

Cet article élabore une nouvelle structure pour permettre d'étudier les jeux (games) comme des lieux de rationalisation sociale, en appliquant la théorie critique de la technologie de Feenberg. Nous commencerons en argumentant que si les jeux (games) sont des systèmes de rationalité sociale, ils doivent être considérés comme étant apparentés à d'autres systèmes modernes, comme les marchés capitalistes et les organisations bureaucratiques. Nous présenterons ensuite une conceptualisation du jeu (play) comme un processus par lequel l'attention du joueur est détournée de l'action indifférenciée de la vie quotidienne vers une sphère différenciée d'activités ludiques. Cette approche révèlera combien l'expérience du jeu (play) change lorsqu'elle est rationalisée par la médiation technologique, et comment une normalisation généralisée se produit quand les jeux (games) deviennent des pratiques sociales à grande échelle. Nous proposerons donc une théorie de la rationalisation du jeu (ludification) décrivant les composants clés des jeux socialement rationalisés; une théorie que nous appliquerons alors à l'exemple spécifique des jeux en ligne massivement multijoueurs (MMOG, pour Massively Multiplayer Online Games).

\section{INDEX}

Mots-clés: Feenberg, jeux rationalisés, ludification, MMOG, rationalité sociale 


\section{AUTHORS}

\section{SARA M. GRIMES}

Communication appliquée et Laboratoire technologique, École de communication, université Simon Fraser, Vancouver, Colombie britannique, Canada.

\section{ANDREW FEENBERG}

Communication appliquée et Laboratoire technologique, École de communication, université Simon Fraser, Vancouver, Colombie britannique, Canada. 